23. vuosik. 1951

\title{
YLEISIMPIEN PUUTARHAKASVIEN SUOMENKIELINEN NIMISTÖ
}

\author{
PUUTARHAVILJELIJÄIN LIITTO r.y:n PUUTARHAKASVIEN NIMISTÖN \\ YHDENMUKAISTAMISTOIMIKUNTA
}

Usealla maassamme viljellyllä puutarhakasvilla on monta suomalaista nimeä, josta aiheutuu sekaannusta. Haitallista on myös, että useammalla kasvilla voi olla sama nimi, joten aina ei voida tietää, mitä kulloinkin nimellä tarkoitetaan. Tämän lisäksi on lukuisia tapauksia, jolloin kasvilla ei ole laisinkaan suomalaista nimeä. Näistä syistä käytetään kasvitieteilijöiden sekä puutarhaviljelijöidenkin keskuudessa yleisimmin kasvien tieteellisiä nimiä. Ei kuitenkaan voida ajatella, että laajat kansalaispiirit opettelisivat kasvien tieteelliset nimet. Niinpä puutarhaviljely on oppiaineena monissa sellaisissa kouluissa, joiden oppilaat eivät ole lukeneet kasvien vieraskielisiä nimiä eivätkä osaa vieraita kieliä. Sellaisia oppilaitoksia ovat esim. lukuisat kotitalousalan koulut ja opistot. Myös eräissä maataloudellisissa oppilaitoksissa kuuluu puutarhaviljely opetusohjelmaan. Tämän lisäksi tulevat monet tuhannet puutarha-alan harrastajat, jotka tarvitsevat puutarhakasvien äidinkielisiä nimiä, mm. esiintyessään siemenkauppojen ja taimistojen asiakkaina.

Edellä esitetyistä syistä asetettiin 28. 2. 1948 toimikunta puutarhakasvien nimistön yhdenmukaistamista varten. Toimikunnassa on ollut Puutarhaviljelijäin Liiton edustajana ja toimikunnan puheenjohtajana Puutarhaopiston johtaja Arvo HaApanen, lisäksi agronomi Jonne Törmä, Kustannusosakeyhtiö Otavan edustajana tohtori Jorma Soveri ja Suomalaisen Eläin- ja Kasvitieteellisen Seuran Vanamon edustajana dosentti Ilmari Hirtonen. Toimikunnan sihteerinä on ollut fil.maisteri, rouva ELVI ERÄMETSÄ. Toimikunnan ensimmäisenä ja tärkeänä tehtävänä oli yleisimpien puutarhakasvien suomenkielisen nimistön laatiminen, josta toimikunta on saanut ehdotuksensa valmiiksi julkaisten sen seuraavassa latinan- ja suomenkielisenä luettelona.

Toimikunta julkaisee puutarhakasvien nimiluettelon siinä mielessä, että suomenkielisiä kasvinnimiä tarvitsevat ryhtyisivät niitä yleisesti käyttämään. Vasta käytössä nimet voivat syöpyä kansalaisten mieliin ja pääsevät yleiseen tietoisuuteen. Erityisen toivottavaa olisi, että maamme taimistot ja siemenkauppiaat alkaisivat käyttää hinnastoissaan ja muussakin liiketoiminnassaan näitä nimiä, koska vakiintunut nimistö on niiden etujen mukaista.

Toimikunta on tietoinen siitä, että sen nimiehdotuksissa saattaa olla paljon sellaisiakin, joita kaikki eivät hyväksy. Sen vuoksi toimikunta toivoo, että sille tehtäisiin huomautuksia ja ehdotuksia, nimiehdotuksia myös sellaisille kasveille, joita luettelossa ei ole mainittu. Jatkuvana työnään toimikunta tulee harkitsemaan tällaisten ehdotusten käyttökelpoisuutta. Kirjeenvaihto osoitetaan Puutarhaviljelijäin Liiton toimistoon, os. Helsinki, Mikonk. 7.

Helsingissä, lokakuun 27 p:nä 1950. 


\section{KASVIEN TIETEELLISTEN NIMIEN LUETTELO}

Useat kansainväliset kongressit ovat suositelleet kasvien tieteellisten lajinimien kirjoittamista poikkeuksettomasti pienin alkukirjaimin. Koska kasvitieteellisen nimistön kansainvälisiin sääntöihin ei ole kuitenkxan vielä sisällytetty nimenomaista määräystä tästä, on seuraavassa luettelossa kirjoitettu suurin alkukirjaimin kuitenkin sellaiset lajien, alalajien ja muunnosten nimet, jotka johtuvat henkilöiden tai paikkakuntien nimistä ja esiintyvät genetiivissä (omannossa).

Ábies - jalokuusi

- álba - Saksan j.

- balsámea - palsamij.

- cóncolor - harmaa j.

- sibírica - Siperian j.

Abútilon - aulio

- hýbridum - vaahtera-a.

- Theophrásti - kelta-a.

Acásia - akaasia

Ácer - vaahtera

- campéstre - niveräv.

- gínnala - Mongolian v.

- negúndo - saarniv.

- platanoídes - metsäv.

- pseudoplátanus - vuoriv.

- saccharínum - hopeav.

- tatáricum - Tatarian v.

Achilléa - kärsämö

— filipendulína - kultak.

- millefólium - siank.

- ptármica - ojak.

- - fl.pl. - tuhatkauno

Achímenes - mustanmerenruusu

Achyránthes $\rightarrow$ Iresine

Aconítum - ukonhattu

- cámmarum - tarhau.

- napéllus - aito $\mathrm{u}$.

Acroclínium $\rightarrow$ Helípterum

Adiántum - hiussaniainen

- capillus-Véneris - Venuksen h.

Adónis - ruusuleinikki

- ánnua - syksyinen r.

- vernális - keväinen r.
Aésculus hippocástanum - hevos- - caudátus - punainen r. kastanja

Agapánthus africánus - sinisarja- ryllis lilja

Amelánchier - tuomipihlaja

Agáve - agaave

Agératum houstoniánum - sinitähtönen

Ájuga - akankaali

— réptans - rönsyinen a.

Állium - sipuli, laukka

- ascalónicum - šalottens.

- cépa - siemens. - spicáta - pienikukkainen $\mathrm{t}$.

Ammóbium alátum - (siipipalteinen) iäisyydenkukka

Amýgdalus $\rightarrow$ Prúnus

Ánanas satívus - ananas

Anchúsa - häränkieli

- itálica - Italian h.

Anemóne - vuokko

- - v. aggregátum - istukass. - apennína - Apenniinien v.

- coerúleum - sinilaukka

- coronária - kruunuv.

- fistulósum - talvis.

- móly - keltalaukka

- pórrum (ampelóprasum) - purjos.

- satívum - valkos.

- schoenóprasum - ruoholaukka

Álnus - leppä

- glutinósa - terval.

- incána - harmaa 1.

Áloë - aaloe

- arboréscens - rohtoa.

- variegáta - kirjava a.

Alsóphila - saniaispuu

Althaéa - salkoruusu

- officinális - rohtos.

- rósea - tarhas.

Alýssum - kilpiruoho ( $\rightarrow$ myös Lobulária)

Amaránthus - revonhäntä
- hupehénsis (sekamuotoineen) syysv.

- silvéstris - arov.

Anéthum gravéolens - tilli

Antennária - kissankäpälä

Ánthemis - sauramo

- tinctória - keltas.

Anthéricum - hietalilja ( $\rightarrow$ myös Paradísia)

Anthríscus cerefólium - maustekirveli

Anthúrium - flamingonkukka

Antirrhínum május - leijonankita

Ápium gravéolen: - ruokaselleri

— - v. dúlce - lehtiselleri

— - v. rapáceum - juuriselleri

Aporocáctus flagellifórmis - käärmekaktus

Aquilégia - akileija 
- chrysántha - kulta-a.

- coerúlea - jalo a.

— vulgáris - lehtoa.

Árabis - pitkäpalko

- alpína - tunturip.

- caucásica (sekamuotoineen) tarhap.

Arália $\rightarrow$ Fátsia

Araucária - araukaaria

- araucána - suomuinen a.

- excélsa - huonekuusi

Arctótis grándis - hopeasilmä

Arenária - arho

Aristolóchia - piippuruoho

- dúrior - köynnösp.

Arméria - laukkaneilikka

- maritima - rannikon 1.

Armorácia rusticána - piparjuuri

Arónia - aronia

Artemísia - maruna

- abrótanum - aaprottim.

- absínthium - koiruoho, mali

Árum maculátum - munkinhuppu

Arúncus silvéster - töyhtöangervo

Aspáragus - parsa

— officinális - ruokaparsa

- plumósus - unelma

- Sprengéri - hienohelma

Aspidístra elátior - tuonenkielo

Asplénium - raunioinen

- bulbíferum - itur.

Áster - asteri

- alpínus - alppia.

- améllus - elokuun a.

- Nóvae-Ángliae - tuoksua.

- Nóvi-Bélgii - syysa.

- subcoerúleus - juhannusa.

Astílbe - jaloangervo

Astrántia - tähtiputki

Átriplex horténsis - tarhamaltsa

Aubriétia deltoídea (sekamuotoineen) - ristikki

Aucúba japónica - kiiltopensas

Azaléa $\rightarrow$ Rhododéndron

Begónia - vinolehti

- cheimántha - annansilmä

- réx (sekamuotoineen) - upea v.

- semperflórens (sekamuotoineen) - ainakukkiva v.

- tuberhýbrida - mukulav.

Béllis perénnis - kaunokainen

Belopérone guttáta - onnenkäpy
Bérberis - happomarja

- Thunbérgii - Japanin h.

- vulgáris - ruosteinen h.

Bergénia crassifólia - vuorenkilpi

Béta vulgáris - juurikas

— - v. cícla - lehtijuurikas

— - v. rúbra - punajuurikas

Betónica - jalopähkämö

Bétula - koivu

- nána - vaivaisk.

- pubéscens - hiesk.

- verrucósa - raudusk.

— - f. dalecárlica - Taalainmaan $\mathrm{k}$.

Billbérgia - papinkaura

- nútans - nuokkuva p.

Boccónia $\rightarrow$ Macleáya

Borágo officinális - purasruoho

Bougainvíllea glábra - ihmeköynnös

Bouvárdia - morsiustähti

Brássica - kaali

- campéstris $\mathrm{v}$. rápa - nauris

- nápus v.napobrássica - lanttu

- olerácea - aito kaali

— - v. acéphala - lehtik.

- - v. botrýtis - kukkak.

— - v. capitáta - keräk.

- - - f. álba - (valkoinen) keräk.

— - - f. rúbra - punakaali

- - v. gemmífera - ruusuk.

— - v. sabáuda - kurttuk.

— - v. gongyloídes - kyssäk.

Browállia - piispankukka

Bryónia - koiranköynnös

- álba - mustamarjainen $\mathrm{k}$.

- dióica - punamarjainen $\mathrm{k}$.

Bryophýllum - itulehti

Búxus sempérvirens - koiranpensas

Caládium - kirjolehti

Calceolária - tohvelikukka

Caléndula officinális - (tavallinen) kehäkukka

Cálla $\rightarrow$ Zantedéschia

Callístephus chinénsis - kiinanasteri

Calystégia sépium - karhunköynnös (iso kierto)

Caméllia japónica - kamelia

Campánula - kellokukka
- carpática - Karpaattien kello

- cochleariifólia - kääpiökello

— frágilis - neidonkello

— isophýlla - morsiuskello

- latifólia - ukonkello

- médium - Maarian kello

- portenschlagiána - rauniok.

Cánna generális - Intian kanna

Cánnabis satíva - hamppu

— — f. gigantéa - jättiläishamppu

Cápsicum ánnuum - turkinpippuri, paprika

Caragána - hernepensas

- arboréscens - Siperian h.

- frútex - Euroopan h.

Cárduus mariánus $\rightarrow$ Sílybum

Carlína - kurho

Carpínus bétulus - valkopyökki

Cárum cárvi - (tavallinen) kumina

Cattléya - katleija

Celástrus - surmaköynnös

Celósia argéntea (cristáta) - kukonharja

Centauréa - kaunokki

- cýanus - ruiskukka (ruiskaunokki)

- dealbáta - huopak.

- montána - vuorik.

- pulchérrima - jalo $\mathrm{k}$.

Cephalária - kirahvinkukka

Cephalocéreus senílis - vaarinkaktus

Cerástium - härkki

- alpínum - tunturih.

- Bieberstéinii - nukkah.

- tomentósum - hopeah.

Céreus - pylväskaktus

Chaenoméles - ruusukvitteni

Chamaecýparis - valesypressi

- lawsoniána - Lawsonin v.

Chamaérops húmilis - kääpiöpalmu

Cheiránthus $\rightarrow$ Erýsimum ja Matthíola

Chionodóxa - kevättähti

Chloróphytum capénse - rönsylilja

Chrysánthemum - päivänkakkara

- carinátum - kirjava p.

- coccíneum - punainen p.

- coronárium - kruunup. 
Chr. frutéscens - pensasp.

- máximum - iso p.

— morifólium - krysanteemi

- parthénium - reunusp.

- ségetum - keltainen p.

— vulgáre - pietaryrtti

Cichórium - sikuri

- endivia - lehtis.

- intybus - juuris.

Cinerária $\rightarrow$ Senécio

Císsus - varjoköynnös

— antárctica - etelän v.

Citrúllus vulgáris - arpuusi

Cítrus - sitruuna

Clárkia - immenkukka

- élegans - komea i.

- pulchélla - hento i.

Clématis - metsäköynnös

- alpína - Siperian m.

- Jackmánii - isokukkainen

— vitálba - Saksan m.

— viticélla - Italian $\mathrm{m}$.

Clerodéndron - kohtalonpensas

Clívia miniáta - punasarja, kliivia

Cobaéa scándens - kelloköynnös

Codiaéum - ihmepensas

Cólchicum autumnále - (tavallinen) myrkkylilja

Cóleus hýbridus - värinokkonen

Colútea arboréscens - rakkopensas

Convallária majális - kielo

Convólvulus - kierto $(\rightarrow$ myös Calystégia)

- trícolor - kolmivärinen $\mathrm{k}$.

Cordylíne - liljapuu

Coreópsis - kaunosilmä

Coriándrum satívum - korianteri

Coronária $\rightarrow$ Lychnis

Córnus - kanukka

- álba - valkomarjainen pensask.

- sanguínea - mustamarjainen pensask.

Corýdalis - kiurunkannus

— nóbilis - komea $\mathrm{k}$.

— sólida - pysty k.

Córylus avellána - pähkinäpensas

Cósmos bipinnátus - kosmoskukka

Cotoneáster - tuhkapensas

- adpréssus - matala $t$.

— integérrimus - punamarjainen $t$.

- lúcidus - kiiltävä $\mathrm{t}$.
- melanocárpus - mustamarjainen $\mathrm{t}$.

Crássula - paunikko, viljellyt lajit - paksulehti

Crataégus - orapihlaja

— "coccinea" - Amerikan o.

- monógyna - tylppäliuskainen o.

— oxyacántha - pyöreäliuskainen o.

Crínum - kriinumi

- Móorei - palmuk.

Crócus - sahrami

- moesíacus - keltas.

— vérnus - keväts.

Cróton $\rightarrow$ Codiaéum

Cúcumis - kurkku

- sativus - (ruoka)k.

- mélo - meloni

. Cucúrbita - kurpitsa

- máxima - jättiläisk.

- pépo - (tavallinen) kurpitsa

Cupréssus sempérvirens - aito sypressi

Cyclámen pérsicum - syklaami

Cydónia oblónga - aito kvitteni

Cymbalária - kilkkaruoho

- murális - rauniok.

- pállida - karvainen k.

Cynára scólymus - latva-artisokka

Cynoglóssum - koirankieli

( $\rightarrow$ myös Omphalódes)

Cynosúrus cristátus - otasukapää

Cypérus - papyruskaisla

Cypripédium - tikankontti

Cýtisus - kultavihma

Dáctylis glomeráta - koiranheinä

Dáhlia cultórum - daalia, syyshehku

Dáphne - näsiä

Datúra - hulluruoho

- stramónium - piikkikotainen h.

- suavéolens - tuoksuva h.

Dáucus caróta - porkkana

Delphínium - ritarinkannus

- Ajácis - etelän r.

- cultórum - jalo r.

- elátum - korkea r.

- orientále - idän r.

Déutzia - nietospensas

Diánthus - neilikka

- barbátus - harjan.
- caryophýllus - tarhan.

- chinénsis - Kiinan n.

- deltoídes - keton.

- plumárius - sulkan.

Dicéntra - särkynyt sydän

- spectábilis - iso särkynyt sydän

Dictámnus álbus - (Mooseksen) palava pensas

Digitális - sormustinkukka

- purpúrea - rohtos.

Dimorphothéca - säänennustaja

- pluviális - valkea s.

- sinuáta - keltainen s.

Dípsacus - karstaohdake

Dodecátheon meádia - jumaltenkukka

Dorónicum - vuohenjuuri

- caucásicum - pieni v.

- excélsum - iso $\mathrm{v}$.

Drába - kynsimö

Dracaéna dráco - lohikäärmeenpuu

Dracocéphalum - ampiaisyrtti

- moldávica - tuoksuva a.

- ruyschiána - isokukkainen a.

- sibíricum - Siperian a.

Drýas - lapinvuokko

Dryópteris fílix-más - kivikon alvejuuri

Echevéria - mehiruusuke

Echinácea purpúrea - punahattu

Echinocáctus - siilikaktus

Echínops - pallo-ohdake

Elaeágnus - hopeapensas

- commutáta - aito h.

Epiphýllum - lehtikaktus ( $\rightarrow$ myös Zygocactus)

Eránthis - talventähti

Eremúrus - marskinlilja

Eríca - kellokanerva

Erígeron speciósum - tarhakallioinen

Erýngium - piikkiputki

Erýsimum chéiri - kultalakka

Eschschóltzia califórnica - tuliunikko

Eucalýptus glóbulus - kuumepuu

Éucharis grandiflóra - Amazonin lilja

Euónymus - sorvarinpensas

- europaéus - Euroopan s.

- japónicus - Japanin s. 
Eupatórium - punalatva

Euphórbia - tyräkki

- cyparíssias - tarhat.

- fúlgens - korallit.

- polýchroma - kultat.

- pulchérrima - joulutähti

— spléndens - piikkikruunu

Exochórda - helmipensas

Fágus silvática - pyökki

Fatshédera Lízei - murattiaraalia

Fátsia japónica - huonearaalia

Festúca - nata

Fícus - viikuna

- cárica - aito viikuna

- elástica - huonekumipuu

- lyráta - lyyralehtinen v.

— púmila - pieni kiipijäv.

— radícans - iso kiipijäv.

Filipéndula - angervo

Foenículum officinále - saksankumina

Forsýthia - onnenpensas

- ováta - Korean onnenpensas

Fragária - mansikka

- ananássa - tarham.

- vésca v. alpína - kuukausim.

Fráxinus - saarni

- excélsior - metsäs.

- pennsylvánica - punas.

- americána - valkos.

Fréesia - runokki

Fritillária - pikarililja

— imperiális - keisarin p.

- meleágris - kirjava p.

Fúchsia - verenpisara

- hýbrida - tavallinen v.

Fúnkia $\rightarrow$ Hósta

Gaillárdia - sädekukka

- aristáta - monivuotinen s.

- hýbrida - yksivuotinen s.

Galánthus nivális - lumikello

Galéga - vuohenherne

Gardénia jasminoídes - keikarinkukka

Genísta - väriherne

- tinctória - keltainen v.

Gentiána - katkero

- acáulis - varreton $\mathrm{k}$.

- lútea - keltak.

- pneumonánthe - keuhkok.

— septémfida - törmäk.
Geránium - kurjenpolvi

Gerbéra Jamesónii - sädelatva

Géum - kellukka

Gília - kiurunkukka

Gladíolus - miekkalilja

- gandavénsis - komea $\mathrm{m}$.

Gloxínia $\rightarrow$ Sinníngia

Godétia - silkkikukka

- amoéna - siro s.

Gypsóphila - raunikki

- élegans - yksivuotinen harsokukka

- paniculáta - morsiamen harsokukka

Hablitzia tamnoídes - köynnöspinaatti

Haemánthus - tupsukki

— álbiflos - valkot.

Hawórthia - kirjotähti

Hédera hélíx - muratti

Helénium - hohdekukka

— autumnále - syysh.

- Hoopésii - korea h.

Heliánthemum - päivännouto

Heliánthus - auringonkukka

- ánnuus - iso auringonkukka

— tuberósus - mukula-artisokka

- rígidus - tankea auringonkukka

Helichrýsum - olkikukka

Heliópsis - päivänsilmä

Heliotrópium - heliotrooppi

Helipterum - ikikukka

- Manglésii - herttalehtinen i.

- róseum - kapealehtinen i.

Helléborus - jouluruusu

- níger - vaalea $\mathrm{j}$.

— víridis - vihertävä j.

Helxíne Soleirólii - kodinonni

Hemerocállis - päivänlilja

- fláva - keltainen p.

- fúlva - punakeltainen p.

Hepática tríloba - sinivuokko

Heracléum - ukonputki

Hésperis matronális - illakko

Heuchéra - keijunkukka

Hibíscus - iibisruusu

- rósa-sinénsis - kiinanruusu

Hierácium - keltano

Hippeástrum hýbridum - ritarinkukka

Hippóphaë rhamnoídes - tyrni
Hósta - kuunlilja

Hówea - kentiapalmu

Hóya carnósa - posliinikukka

Húmulus lúpulus - humala

Hyacínthus orientális - hyasintti

Hydrangéa - hortensia

- macrophýlla - isokukkainen $\mathrm{h}$.

- paniculáta - syreenih.

Hymenocállis - lukinlilja

Hyssópus officinális - iisoppi

Ibéris - saippo

- amára - katkera saippo

- sempérvirens - talvisaippo

- umbelláta - sarjasaippo

flex aquifólium - orjanlaakeri

Impátiens - häpykannus

- balsámina - palsami

- glandulígera - jättiläispalsami

- Hólstii ja I. sultáni (sekamuotoineen) - ahkeraliisa

Incarvíllea - lähetyssaarnaajankukka

Ínula helénium - iso hirvenjuuri Ipomoéa - elämänlanka

- purpúrea - aito e.

Iresíne - punalehti

Íris - kurjenmiekka

- germánica - Saksan k.

- sibírica - Siperian k.

Jásminum - jasmiini

- grandiflórum - aito jasmiini

- odoratíssimum - tuoksujasmiini

Júglans - jalopähkinä

- cinérea - Amerikan j.

- mandschúrica - Mantšuurian j.

Juníperus - kataja

- commúnis - tavallinen $\mathrm{k}$.

- sabína - rohtok.

- virginiána - kynäk.

Kalánchoë blossfeldiána - tulilatva Kéntia $\rightarrow$ Hówea

Kléinia répens - mehivillakko

Kniphófia - soihtulilja

Kóchia scopária - kesäsypressi

Labúrnum - kultasade

- alpínum - kalju $\mathrm{k}$.

- anagyroídes - karvainen $\mathrm{k}$. 
Lactúca - salaatti

- satíva - ruokasalaatti

— - v. capitáta - keräs.

— - v. foliósa - lehtis.

— - v. romána - sides.

Lárix - lehtikuusi

- decídua - Euroopan 1.

- sibírica - Siperian 1.

Latánia $\rightarrow$ Livistóna

Láthyrus - nätkelmä

- odorátus - tuoksuherne

Láurus nóbilis - jalolaakeri

Lavándula officinális - laventeli

Lavatéra - poppeliruusu

- thuringíaca - harmaa p.

- triméstris - kolmikuinen p.

Leontopódium alpínum - alppitähti

Lepídium satívum - vihanneskrassi

Leucójum vérnum - kevätkello

Levísticum officinále - rohtoliperi

Liátris - punatähkä

Ligulária - nauhus

Ligústrum - likusteri

— vulgáre - aital.

Lílium - lilja

- bulbíferum - ruskol. (keisarinkruunu)

- cándidum - Maarian 1.

- mártagon - varjol.

- regále - kuningasl.

- tigrínum - tiikeril.

Limónium - ikiviuhko

Linária - kannusruoho

Línum grandiflórum - punapellava

Livistóna chinénsis - viuhkapalmu

Lobélia - nuottaruoho, elvikki

- érinus - sinielvikki

— fúlgens ja L. spléndens - punaelvikki

Lobulária marítima - valkopielus

Lólium - raiheinä

- perénne - Englannin r.

- multiflórum - Italian r.

Lonicéra - kuusama

- caprifólium - aito köynnösk.

- japónica v. áureo-reticuláta kirjokuusama

- periclýmenum - Ruotsin köynnösk.
- tatárica - rusokuusama

- xylósteum - lehtokuusama

Lúffa - pesusienikurkku

Lunária - kuuruoho

Lupínus - lupiini

- hýbridus - yksivuotinen 1 .

- polyphýllus - komea 1 .

Lýchnis - käenkukka

- chalcedónica - palava rakkaus

- flós-Jovis - Juppiterin kukka

Lysimáchia - alpi

- nummulária - suikeroa.

Lýthrum - rantakukka

- salicária - tavallinen $r$.

— virgátum - Amerikan r.

Macleáya cordáta - röyhyunikko

Mahónia aquifólium - mahonia

Majorána horténsis - maustemeirami

Málope trífida - maloppi

Málus - omenapuu, omena

- baccáta - marjao.

- doméstica - tavallinen o.

- prunifólia - tuomenlehtinen o.

- purpúrea - purppurao.

- silvéstris - metsäo.

Málva - malva

- álcea - ruusum.

- moscháta - myskim.

Malvástrum capénse - huonemalva

Mammillária - syyläkaktus

Maránta - nukkumatti

Mattéuccia struthiópteris - kotkansiipi

Matthíola - leukoija

- bicórnis - sarvil.

- incána - tarhal.

Medicágo - mailanen

Méntha - minttu

- piperíta - piparm.

Mesembryánthemum - päivikki

- crystállinum - jääruoho

- conspícuum - helopäivikki

Méspilus germánica - (Saksan) mispeli

Mikánia $\rightarrow$ Senécio

Mimósa pudíca - tuntokasvi

Mímulus - apinankukka

- guttátus - keltainen a.

— tigrínus - täplikäs a.
Minuártia - nätä

Mirábilis - ihmekukka

- jalápa - tuoksuton i.

Monárda - hevosminttu

Monstéra deliciósa - peikonlehtı

Montbrétia $\rightarrow$ Tritónia

Mórus - silkkiäispuu

Músa - banaani ,

Muscári - helmililja

- botryoídes - hento $\mathrm{h}$.

Myosótis - lemmikki

- silvática - lehtol.

Mýrrhis odoráta - saksankirveli

Mýrtus commúnis - myrtti

— - v. microphýlla - morsiusm.

Narcíssus - narsissi

- poëticus - valkon.

- pseudonarcíssus - keltan.

- tazétta - sarjan.

Nemésia - kostonkukka

Nemóphila - sievikki

- Menziésii - sinis.

Népeta - kissanminttu

- pseudo-Mussínii - matala $\mathrm{k}$.

Nephrólepis exaltáta - sulkasaniainen

Nérium oleánder - aito oleanteri

Nertéra granadénsis - korallimätäs

Nicotiána - tupakka

— aláta - koristet.

- rústica - palturit.

- tabácum - Virginian $\mathrm{t}$.

Nigélla - neidonkukka

- damascéna - kesäinen n.

Nymphaéa - lumme

- álba - iso 1.

- cándida - tavallinen 1 .

- tetrágona - pikkul.

Oenothéra - helokki

Omphalódes - ikävänkukka

- linifólia - kapealehtinen i.

- vérna - leveälehtinen i.

Onóclea $\rightarrow$ Mattéuccia

Onopórdum acánthium - kruunuohdake

Ophiopógon - käärmeenparta

Opúntia - opuntiakaktus

- fícus-índica - viikunakaktus

Orchis - kämmekkä

Oríganum majorána $\rightarrow$ Majorána 
Ornithógalum - linnunmaito

Óxalis - käenkaali

Paeónia - pioni

- festíva - talonpoikaisp.

- lactiflóra - jalo p.

- tenuifólia - hienoliuskainen p.

Papáver - unikko

- alpínum - alppiu.

— bracteátum $\rightarrow$ P. orientále

- gláucum - tulppaaniu.

— nudicáule - Siperian u.

— orientále - idän u.

— rhoéas - silkkiu.

— somníferum - oopiumiu.

Paradísia liliástrum - paratiisinlilja

Parthenocíssus - villiviini

- vitácea - tavallinen v.

- quinquefólia (Engelmánii) imukärhinen v.

Passiflóra - kärsimyskukka

- coerúlea - sininen $\mathrm{k}$.

Pastináca satíva - palsternakka

Pelargónium - pielikukka, pelargoni

— domésticum - jalo p.

- hortórum - tavallinen p.

— peltátum - riippap.

Pentstémon barbátus - punainen kilpikukka

Peperómia - muorinkukka

Perílla frutéscens - veripeippi

Petasítes - ruttojuuri

Petroselínum horténse - persilja

Petúnia hýbrida - petunia

Phacélia - hunajakukka

- tanacetifólia - aito h.

Phálaris arundinácea - ruokohelpi

Phaséolus - papu

- coccíneus - ruusup.

- vulgáris - tarhap.

— - v. commúnis - salkop.

— - v. nánus - pensasp.

Philadélphus - pihajasmiini

- coronárius - tavallinen p.

- grandiflórus - isokukkainen p.

- Lemoínei - Ranskan p.

— virginális - kameliankukkainen p.

Philodéndron - köynnösvehka

( $\rightarrow$ myös Monstéra)
Phléum praténse - timotei

Phlóx - leimukukka

— Drummóndii - kesäl.

- glabérrima (suffruticósa) kiiltolehtinen 1.

- paniculáta - syysl.

— stolonífera - rönsyl.

— subuláta - sammall.

Phoénix - taatelipalmu

Phyllítis scolopéndrium - hirvenkieli

Phyllocáctus $\rightarrow$ Epiphýllum

Phýsalis alkekéngi - juutalaiskirsikka

- bistórta - konnant.

- cuspidátum - Japanin $\mathrm{t}$.

— sachalinénse - jättiläist.

Pópulus - haapa, poppeli

- álba - hopeap.

- balsamífera - palsamip.

- laurifólia - laakerip.

- nígra - mustap.

- trémula - haapa

— trichocárpa - jättiläisp.

Portuláca - portulakka

- grandiflóra - koristep.

- olerácea - vihannesp.

Potentílla - hanhikki

Physocárpus opulifólius - heisi- — atrosanguínea - verih. angervo - fruticósa - pensash.

Pícea - kuusi - hýbrida - punah.

- ábies (excélsa) - tavallinen k., - nepalénsis - Intian h. näre Prímula - esikko

- Engelmánii - Engelmanin k. - aurícula - kultae.

— gláuca (canadénsis) - valkok. - cortusoídes - purppurae.

- omórika - Serbian k. - denticuláta - palloe.

- púngens - okak. - elátior - etelän keväte.

— - f. argéntea ja f. gláuca - — farinósa - jauhoe.

hopeaokak.

Pimpinélla - pukinjuuri

- anísum - anisruoho

Pínus - mänty

- cémbra - sembram.

- múgo (montána) - vuorim.

- péuce - Makedonian $\mathrm{m}$.

- silvéstris - tavallinen $\mathrm{m}$., petäjä

— stróbus - strobusm.

Pírus $\rightarrow$ Málus ja Pýrus

Písum satívum - kylvöherne

— - ssp. arvénse - peltoherne

- - ssp. horténse - tarhaherne

Platycérium - hirvensarvisaniainen

Platycódon grandiflórus - jalokello

Plectránthus Oertendáhlii - liisankukka

Plumbágo capénsis - lyijykukka

Póa - nurmikka

Poinséttia $\rightarrow$ Euphórbia pulchérrima

Polemónium coerúleum - lehtosinilatva

Polygónatum - kalliokielo

Polýgonum - tatar

- alpínum - alppit.
- Floríndae - kesäe.

- malacoídes - malvae.

- obcónica - huone-e.

- sinénsis - Kiinan e.

— véris - keväte.

Prúnus - tuomi

— amýgdalus - manteli

— armeníaca - aprikoosi

— ávium - imelä kirsikka

- cérasus - hapan kirsikka

- doméstica - luumupuu, luumu

- - v. insitítia - kriikunapuu, kriikuna

— Máackii - tuohituomi

— pádus - tavallinen tuomi

- pérsica - persikka

— serótina - kiiltotuomi

- spinósa - oratuomi

— tenélla - kääpiömanteli

— tríloba - ruusumanteli

- virginiána - Virginian tuomi

Pseudotsúga taxifólia - douglaskuusi

Pulmonária - imikkä

Pulsátilla - kylmänkukka

- alpína - alppien $\mathrm{k}$.

- montána - vuorten $\mathrm{k}$.

— vernális - kangasvuokko

— vulgáris - lännen $\mathrm{k}$. 
Púnica granátum - granaattiomena

— - v. nána - kääpiög.

Pýrethrum $\rightarrow$ Chrysánthemum

Pýrus commúnis - päärynäpuu, päärynä

Quércus - tammi

— róbur - (kesä)tammi

- boreális v. máxima - punat.

- petraéa - talvit.

Ranúnculus - leinikki

Ráphanus satívus - ruokaretikka - — v. radícula - retiisi

Reséda - reseda

- odoráta - tuoksur.

Rhámnus - paatsama

- cathártica - orap.

Rhéum - raparperi

- palmátum - korister.

- rhapónticum - ruokar.

Rhípsalis - korallikaktus

Rhodánthe $\rightarrow$ Helípterum

Rhododéndron - alppiruusu

- catawbiénse - puistoa.

- japónicum - loimua.

- Símsii (Azaléa índica) - atsalea(-alppiruusu)

Rhoicíssus rhomboídea - kuningasviini

Ríbes - herukka

- alpínum - taikinamarja

- áureum - kultah.

- nígrum - mustah.

- rúbrum - punah.

- úva-críspa (grossulária) - karviainen

Rícinus commúnis - risiini

Rósa - ruusu

- canína - koiranr.

- chinénsis - Bengalin r.

- foétida (lútea) - Persian keltar.

- gállica - Ranskan r.

_ — v. spléndens - Valamon r.

- - ssp. centifólia - kartanonr.

- gláuca - orjanr.

- odoráta - teer.

- pimpinellifólia - juhannusr.

- polyántha - tertturuusu

- rubrifólia - punalehtinen r.

- rugósa - kurttulehtinen r.

— villósa v. pomífera - luumur.
Rosmarínus officinális - ros- Scílla - sinililja mariini

Rúbus - vatukka

- idaéus - vattu

- odorátus - tuoksuvatukka

Rudbéckia - päivänhattu

- laciniáta - kultap.

Rúmex patiéntia - pinaattihierakka

Rúscus aculeátus - rautamyrtti

Rúta gravéolens - ruuta

Sagína horténsis - tarhahaarikko Saintpáulia ionántha - Paavalin kukka

Sálix - paju

- álba - hopeap.

- cáprea - raita

— daphnoídes (ja - härmäp.

— frágilis - piilipuu

- pentándra - halava

- purpúrea - punapaju

- viminális - koripaju

Salpiglóssis sinuáta - torvikukka

Sálvia - salvia

— argéntea - hopeasalvia

- spléndens - tulisalvia

Salvínia rotundifólia - kellussaniainen

Sambúcus - selja

— nígra - mustas.

- racemósa - terttus.

Sanguisórba - punaluppu

Sanseviéra trifasciáta - pistinruoho

Santolína chamaecyparíssus - hopeayrtti

Saponária officinális - suopayrtti

Saturéja - käenminttu

- horténsis - (tarha)kynteli

Saxífraga - rikko, kivirikko

- aizóon - hopear.

- caespitósa (ja S. decípiens) mätäsr.

- cotylédon - tunturir.

- hypnoídes - sammalr.

- sarmentósa - aaroninparta

Scabiósa - törmäkukka

- atropurpúrea - korea t.

- caucásica - Kaukasian t.

Schizánthus - perhoskukka
- sibírica - idän s.

Scindápsus áureus - kultaköynnös

Scolopéndrium $\rightarrow$ Phyllítis

Scorzonéra hispánica - mustajuuri

Sédum - maksaruoho

- ácre - katkera m.

- aizóon - Siperian $\mathrm{m}$.

- álbum - valkea m.

- Ewérsii - Turkestanin $\mathrm{m}$.

- lýdium - Lyydian m.

- rupéstre - kalliom.

- Siebóldii - amppelim.

- spúrium - Kaukasian m.

Selaginélla - mähkä

Selenicéreus - yökaktus

- grandiflórus - yön kuningatar

Sempervívum - mehitähti

- arachnoídeum - seittim.

— sobolíferum - keltainen $\mathrm{m}$.

- tectórum - kattom.

Senécio - villakko ( $\rightarrow$ myös Kléinia ja Ligulária)

- cinerária - tuhkaruoho

- cruéntus - sineraaria

- mikanioídes - murattivillakko

Sidálcea - jalomalva

Siléne - kohokki

— acáulis - tunturik.

- alpéstris - alppik.

— scháfta - syysk.

Sílphium - kompassikukka

Sílybum mariánum - maarianohdake

Sinápis álba - keltasinappi

Sinníngia speciósa - suppilokukka

Solánum - koiso

- capsicástrum - huonekoiso

- dulcamára - punakoiso

- lycopérsicum - tomaatti

- melongéna - munakoiso

- tuberósum - peruna

Soldanélla alpína - alppikello

Solidágo - piisku

- canadénsis - Kanadan p.

- hýbrida - tarhap.

- rugósa - töyhtöp.

Sorbária sorbifólia - pihlaja-angervo

Sórbus - pihlaja

- aucupária - kotip.

- hýbrida - Suomen p.

- intermédia - Ruotsin p. 
Sparmánnia africána - huoneleh- Thalíctrum - ängelmä mus

Spinácia olerácea - aito pinaatti

Spiraéa - pensasangervo $(\rightarrow$ myös Arúncus, Astílbe, Filipéndula, Physocárpus ja Sorbária)

- argúta - morsiusangervo

- chamaedryfólia - virpiangervo

- japónica (callósa) - Japanin p.

- salicifólia - pajunlehtiangervo

- trilobáta - Siperian pensasa.

- Vanhóuttei - kinosangervo

Stáchys - pähkämö $(\rightarrow$ myös Betónica)

- olýmpica (lanáta) - nukkap.

Stapélia - raatokukka

Státice $\rightarrow$ Arméria, Limónium

Stephanótis floribúnda - tuoksuköynnös

Stípa pennáta - höyhenheinä

Strelítzia regínae - kolibrinkukka

Streptocárpus - soilikki

Struthiópteris $\rightarrow$ Mattéuccia

Symphoricárpos rivuláris (racemósus) - lumimarja

Syrínga - syreeni

- josikaéa - Unkarin s.

-- refléxa - nuokkus.

- Wólfii - Korean s.

- vulgáris - pihas.

Tagétes - samettikukka

- erécta - isomykeröinen $\mathrm{s}$.

- pátula - ryhmäs.

Tanacétum $\rightarrow$ Chrysánthemum vulgáre

Táxus - marjakuusi

- baccáta - Europan m.

- cuspidáta - Japanin m.

Tetragónia expánsa - lamopinaatti

Tetragonólobus purpúreus - parsaherne

- Delawáyi - jalo ä. pikkuä.

Thúja - tuija

Thýmus - ajuruoho

- vulgáris - tarha-a.

Tília - lehmus

— vulgáris - puistol. $(\rightarrow$ myös Zebrína)

Trifólium - apila

- répens - valkoa.

Tritóma $\rightarrow$ Kniphófia

Tróllius - kullero

- hýbridus - tarhak.

- május - iso $\mathrm{k}$.

Tsúga - hemlokki

Túlipa - tulppaani

Týpha - osmankäämi

Ûlmus - jalava

- glábra - vuorij.
— aquilegiifólium - lehtoä.

— mínus (ja T. adiantifólium) - Valeriána - virmajuuri

- occidentális - Kanadan t.

Thujópsis dolabráta - hiba-tuija

Thunbérgia aláta - mustasilmä

- pseudolanuginósus - harmaa a.

- serpýllum - kangasa.

- cordáta - niinipuu (metsäl.).

- platyphýlla - isolehtinen 1.

- tomentósa - hopeal.

Tradescántia - kolmiokukka

- virginiána - Virginian $\mathrm{k}$.

Tragopógon - pukinparta

- porrifólius - kaurajuuri

- hýbridum - alsikeapila

- praténse - puna-apila

Tritónia crocosmiiflóra - syyslilja

- europaéus - niittyk.

Tropaéolum - koristekrassi

- peregrínum - siro $\mathrm{k}$.

- gesneriána - tarhat.
- laévis - kynneppää(jalava), kynäj.

Vallóta speciósa - vaarinkukka

Velthéimia capénsis - heimikki

Verátrum - pärskäjuuri

Verbáscum - tulikukka

Verbéna - rautayrtti

Verónica - tädyke

Vibúrnum - heisi

- Carlésii - tuoksuh.

- lantána - villah.

- ópulus - koiran h.

— _ f. roseum - lumipalloh.

Vícia - virna

- fába - härkäpapu

Vínca mínor - talvio

Víola - orvokki

- cornúta - sarvio.

- odoráta - tuoksuo.

— wittrockiána - jättiläiso.

Vítis - viiniköynnös

- labrúsca - ojukkav.

- vinífera - aito $\mathrm{v}$.

Xeránthemum ánnuum - paperikukka

Yúcca - palmulilja

Zantedéschia - huonevehka

- aethiópica - valkoinen $\mathrm{h}$.

- elliottiána - keltainen h.

Zéa máys - maissi

Zebrína péndula - juorukki

Zephyránthes - heinälilja

- cándida - valkea h.

- grandiflóra - punainen $\mathrm{h}$.

Zínnia élegans - oppineittenkukka

Zygocáctus truncátus - joulukaktus 


\section{KASVIEN SUOMENKIELISTEN NIMIEN MUKAINEN HAKEMISTO}

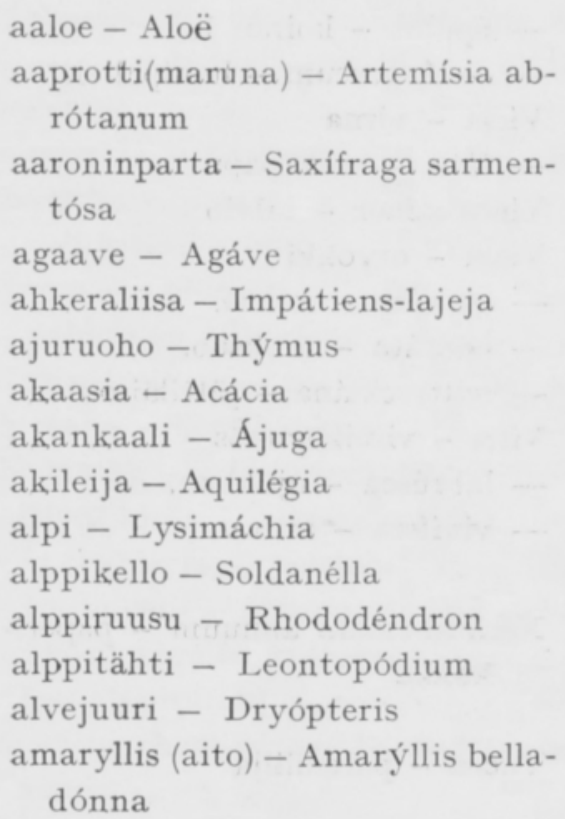

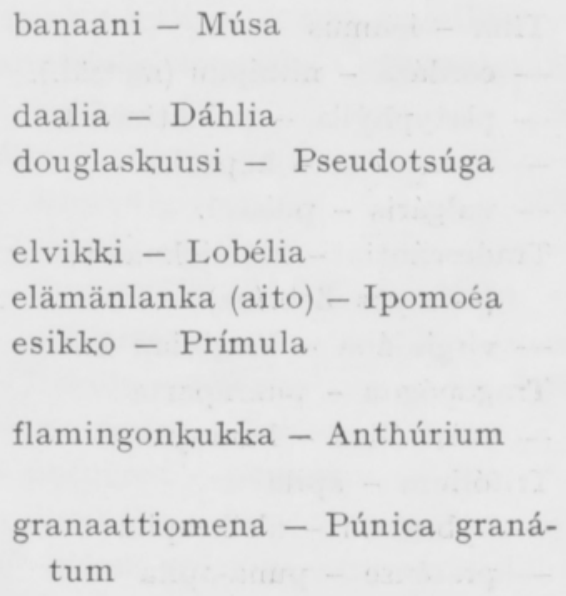

hierakka - Rúmex

hietalilja - Anthéricum

hirvenjuuri - Ínula

hirvenkieli - Phyllítis

hirvensarvisaniainen - Platycérium

hiussaniainen - Adiántum

hohdekukka - Helénium

hopeapensas - Elaeágnus

hopeasilmä - Arctótis

hopeayrtti - Santolína

hortensia - Hydrangéa

hulluruoho - Datúra

humala - Húmulus

hunajakukka - Phacélia

huonearaalia - Fátsia

huonekumipuu - Fícus elástica huonekuusi - Araucária excélsa

huonelehmus - Sparmánnia

huonemalva - Malvástrum

huonevehka - Zantedéschia

hyasintti - Hyacínthus

häpykannus - Impátiens

härkki - Cérastium

härkäpapu - Vícia fába

häränkieli - Anchúsa

höyhenheinä - Stípa

ihmekukka - Mirábilis

ihmeköynnös - Bougainvíllea

ihmepensas - Codiaéum

iibisruusu - Hibíscus

iisoppi - Hyssópus

ikikukka - Helípterum

ikiviuhko - Limónium

ikävänkukka - Omphalódes

illakko - Hésperis

imikkä - Pulmonária

immenkukka - Clárkia

itulehti - Bryophýllum 
iäisyydenkukka - Ammóbium; keijunkukka-Heuchéra myös $\rightarrow$ ikikukka

jalava - Úlmus

jaloangervo - Astílbe

jalokello - Platycódon

jalokuusi - Ábies

jalolaakeri - Láurus nóbilis

jalomalva - Sidálcea

jalopähkinä - Júglans

jalopähkämö - Betónica

jasmiini - Jásminum; myös

$\rightarrow$ pihaj.

joulukaktus - Zygocáctus

jouluruusu - Helléborus

joulutähti - Euphórbia pulchérrima

jumaltenkukka - Dodecátheon

juorukki - Zebrína

Juppiterin kukka - Lychnis flósJovis

juurikas - Béta

juutalaiskirsikka - Phýsalis

jääruoho - Mesembryánthemum crystállinum

kaali - Brássica; myös $\rightarrow$ akank., käenk.

kaktus $\rightarrow$ jouluk., korallik., käärmek., lehtik., opuntiak., pylväsk., siilik., syyläk., vaarink., viikunak., yök.

kallioinen - Erígeron

kalliokielo - Polygónatum

kamelia - Caméllia

kangasvuokko - Pulsatílla vernális

kanna - Cánna

kannusruoho - Linária

kanukka - Córnus

karhunköynnös - Calystégia

karstaohdake - Dípsacus

karviainen - Ríbes úva-críspa

kataja - Juníperus

katkero - Gentiána

katleija - Cattléya

kaunokainen - Béllis

kaunokki - Centauréa

kaunosilmä - Coreópsis

kaurajuuri - Tragopógon porrifólius

kehäkukka - Caléndula keikarinkukka - Gardénia

keisarinkruunu - Lílium bulbíferum

keilo - Campánula; myös

$\rightarrow$ alppik., jaìk., kevätk., lumik.

kellokanerva - Eríca

kellokukka - Campánula

kelloköynnös - Cobaéa

kellukka - Géum

kellussaniainen - Salvínia

keltano - Hierácium

kentiapalmu - Hówea

kesäsypressi - Kóchia

kevätkello - Leucójum

kevättähti - Chionodóxa

kielo - Convallária; myös

$\rightarrow$ kalliok., tuonenk.

kierto - Convólvulus; myös

$\rightarrow$ Calystégia

kiiltopensas - Aucúba

kiinanasteri - Callístephus

kiinanruusu - Hibíscus rósasinénsis

kilkkaruoho - Cymbalária

kilpikukka - Pentstémon

kilpiruoho - Alýssum

kineraaria $\rightarrow$ sineraaria

kirahvinkukka - Cephalária

kirjolehti - Caládium

kirjotähti - Hawórthia

kirsikka - Prúnus-lajeja; myös

$\rightarrow$ juutalaisk.

kirveli $\rightarrow$ maustek., saksank.

kissankäpälä - Antennária

kissanminttu - Népeta

kiurunkannus - Corýdalis

kivirikko - Saxífraga

kliivia $\rightarrow$ punasarja

kodinonni - Helxíne

kohokki - Siléne

kohtalonpensas - Clerodéndron

koiranheinä - Dáctylis

koirankieli - Cynoglóssum

koiranköynnös - Bryónia

koiranpensas - Búxus

koiruoho - Artemísia absínthium

koiso - Solánum

koivu - Bétula

kolibrinkukka - Strelítzia

kolmiokukka - Tradescántia

kompassikukka - Sílphium

korallikaktus - Rhípsalis korallimätäs - Nertéra

korianteri - Coriándrum

koristekrassi - Tropaéolum

kosmoskukka - Cósmos

kostonkukka - Nemésia

kotkansiipi - Mattéuccia

krassi - Lepidium; myös $\rightarrow$ koristek.

kriikuna(puu) - Prúnus domés-

tica v. insitítia

kriinumi - Crínum

kruunuohdake - Onopórdum

krysanteemi - Chrysánthemum morifólium

kukonharja - Celósia

kullero - Tróllius

kultaköynnös - Scindápsus

kultalakła - Erýsimum chéiri

kultasade - Labúrnum

kultavihma - Cýtisus

kumina - Cárum; myös $\rightarrow$ saksank.

kumipuu $\rightarrow$ huonek.

kuningasviini - Rhoicíssus

kurho - Carlína

kurjenmiekka - Íris

kurjenpolvi - Geránium

kurkku - Cúcumis; myös $\rightarrow$ pesusienik.

kurpitsa - Cucúrbita

kuumepuu - Eucalýptus glóbulus

kuunlilja - Hósta

kuuruoho - Lunária

kuusama - Lonicéra

kuusi - Pícea; myös douglask.,

huonek., jalok., lehtik., marjak.

kvitteni (aito) - Cydónia; myös

$\rightarrow$ ruusukv.

kylmänkukka - Pulsatílla

kylvöherne - Písum

kynneppää(jalava) - Ưlmus

laévis

kynsimö - Drába

kynteli - Saturéja horténsis

käenkaali - Óxalis

käenkukka - Lýchnis

käenminttu - Saturéja

kämmekkä - Orchis

kärsimyskukka - Passiflóra

kärsämö - Achilléa

kääpiöpalmu - Chamaérops

käärmeenparta - Ophiopógon

käärmekaktus - Aporocáctus 
köynnöskuusama - Lonicéra-

lajeja

köynnöspinaatti - Hablítzia

köynnösvehka - Philodéndron

laakeri $\rightarrow$ jalol, , orjanl.

lamopinaatti - Tetragónia

lanttu - Brássica nápus

lapinvuokko - Drýas

latva-artisokka - Cynára

laukka - Állium

laukkaneilikka - Arméria

laventeli - Lavéndula

lehmus - Tília; myös $\rightarrow$ huonel.

lehtikaktus - Epiphýllum

lehtikuusi - Lárix

leijonankita - Antirrhínum

leimukukka - Phlox

leinikki - Ranúnculus; myös

$\rightarrow$ ruusul.

lemmikki - Myosótis

leppä - Álnus

leukoija - Matthíola

liisankukka - Plectránthus

likusteri - Ligústrum

lilja - Lílium; myös $\rightarrow$ Amazonin

1., heinäl., helmil., hietal., kuunl., lukinl., marskinl., miekkal., myrkkyl., palmul., paratiisinl., pikaril., päivänl., rönsyl., sinil., sinisarjal., soihtul., syysl.

liljapuu - Cordylíne

linnunmaito - Ornithógalum

liperi - Levísticum

lohikäärmeenpuu - Dracaéna

lukinlilja - Hymenocállis

lumikello - Galánthus

lumimarja - Symphoricárpos

lumme - Nymphaéa

lupiini - Lupínus

luumu(puu) - Prúnus doméstica

lyijykukka - Plumbágo

lähetyssaarnaajankukka - Incarvíllea

maarianohdake - Sílybum

mahonia - Mahónia

mailanen - Medicágo

maissi - Zéa

maksaruoho - Sédum

mali - Artemísia absínthium

malcppi - Málope

maltsa - Átriplex malva - Málva; myös $\rightarrow$ huonem., nätkelmä - Láthyrus

jalom.

mansikka - Fragária

manteli - Prúnus-lajeja

marjakuusi - Táxus

marskinlilja - Eremúrus

maruna - Artemísia

maustekirveli - Anthríscus cere-

fólium

maustemeirami - Majorána

mehiruusuke - Echevéria

mehitähti - Sempervívum

mehivillakko - Kléinia

meirami $\rightarrow$ maustem.

meloni - Cúcumis mélo

metsäköynnös - Clématis

miekkalilja - Gladíolus

minttu - Méntha; myös $\rightarrow$ he-

vosm., kissanm., käenm.

mispeli - Méspilus

Mooseksen palava pensas - Dictámnus

morsiustähti - Bouvárdia

mukula-artisokka - Heliánthus tuberósus

munkinhuppu - Árum

muorinkukka - Peperómia

muratti - Hédera

murattiaraalia - Fatshédera

mustajuuri - Scorzonéra hispánica

mustanmerenruusu - Achímenes mustasilmä - Thunbérgia

myrkkylilja - Cólchicum

myrtti-Mýrtus; myös $\rightarrow$ rautam.

mähkä - Selaginélla

mänty - Pínus

narsissi - Narcíssus

nata - Festúca

nauhus - Ligulária

nauris - Brassica campéstris

v. rápa

neidonkukka - Nigélla

neilikka - Diánthus; myös

$\rightarrow$ laukkan.

nietospensas - Déutzia

niinipuu - Tília cordáta

nukkumatti - Maránta

nuottaruoho $\rightarrow$ elvikki

nurmikka - Póa

näre - Pícea ábies

näsiä - Dáphne nätä - Minuártia

ohdake $\rightarrow$ karstao., kruunuo., maariano. pallo-o.

oleanteri - Nérium oleánder olkikukka - Helichrýsum omena(puu) - Málus; myös

$\rightarrow$ granaattio.

onnenkäpy - Belopérone onnenpensas - Forsýthia oppineittenkukka - Zínnia opuntiakaktus - Opúntia orapihlaja - Crataégus orjanlaakeri - Ílex orvokki - Víola osmankäämi - Týpha

paatsama - Rhámnus

Paavalin kukka - Saintpáulia

paju - Sálix

paksulehti - Crássula

palava pensas - Dictámnus

palava rakkaus - Lýchnis chalce-

dónica

pallo-ohdake - Echínops

palmu $\rightarrow$ kentiap., kääpiöp., taa-

telip., viuhkap.

palmulilja - Yúcca

palsami - Impátiens-lajeja

palsternakka - Pastináca

paperikukka - Xeránthemum

papinkaura - Billbérgia

paprika - Cápsicum ánnuum

papu - Phaséolus; myös $\rightarrow$ här-

käp.

papyruskaisla - Cypérus

paratiisinlilja - Paradísia

parsa - Aspáragus

parsaherne - Tetragonólobus

paunikko $\rightarrow$ paksulehti

peikonlehti - Monstéra

pelargoni - Pelargónium

pellava - Línum

pensasangervo - Spiraéa

perhoskukka - Schizánthus

persikka - Prúnus pérsica

persilja - Petroselínum

peruna - Solánum tuberósum

pesusienikurkku - Lúffa

petunia - Petúnia

petäjä - Pínus silvéstris

pielikukka - Pelargónium 
pietaryrtti - Chrysánthemum vulgáre

pihajasmiini - Philadélphus

pihlaja - Sórbus; myös $\rightarrow$ orap., tuomip.

pihlaja-angervo - Sorbária

fiikkikruunu - Euphórbia spléndens

piikkiputki - Erýngium

piilipuu - Sálix frágilis

piippuruoho - Arıstolóchia

piisku - Solidágo

piispankukka - Browállia

pikarililja - Fritillária

pinaatti (aito) - Spinácia; myös

$\rightarrow$ köynnösp., lamop.

pinaattihierakka - Rúmex patiéntia

pioni - Paeónia

piparjuuri - Armorácia

pistinruoho - Sanseviéra

pitkäpalko - Árabis

poppeli - Pópulus

poppeliruusu - Lavatéra

porkkana - Dáucus

portulakka - Portuláca

posliinikukka - Hóya

pukinjuuri - Pimpinélla

pukinparta - Tragopógon

punahattu - Echinácea

punalatva - Eupatórium

punalehti - Iresíne

punaluppu - Sanguisórba

punasarja - Clívia

punatähkä - Liátris

purasruoho - Borágo

purjo(sipuli) - Állium pórrum

puulilja $\rightarrow$ liljapuu

pylväskaktus - Céreus

pyökki - Fágus; myös $\rightarrow$ valkop. pähkinä $\rightarrow$ jalop., pähkinäpensas pähkinäpensas - Córylus

pähkämö - Stáchys; myös

$\rightarrow$ jalop.

päivikki - Mesembryánthemum päivänhattu - Rudbéckia päivänkakkara - Chrysánthemum

päivänlilja - Hemerocállis päivännouto - Heliánthemum

päivänsilmä - Heliópsis

pärskäjuuri - Verátrum

päärynä(puu) - Pýrus commúnis raatokukka - Stapélia

raiheinä - Lólium

raita - Sálix cáprea

rakkopensas - Colútea.

rantakukka - Lýthrum

raparperi - Rhéum

raunikki - Gypsóphila

raunioinen - Asplénium

rautamyrtti - Rúscus

rautayrtti - Verbéna

reseda - Reséda

retiisi - Ráphanus satívus v. ra-

dícula

retikka - Ráphanus

revonhäntä - Amaránthus

rikko - Saxífraga

risiini - Rícinus

ristikki - Aubriétia

ritarinkannus - Delphinium

ritarinkukka - Hippeástrum

rohtoliperi - Levísticum

rosmariini - Rosmarínus

ruiskukka - Centauréa cýanus

runokki - Fréesia

ruokaselleri - Ápium

ruttojuuri - Petasites

ruusu - Rósa; myös $\rightarrow$ alppir.,

iibisr., joulur., kiinanr., mus-

tanmerenr., poppelir., salkor.

ruusukvitteni - Chaenoméles

ruusuleinikki - Adónis

ruuta - Rúta

rönsylilja - Chloróphytum

röyhyunikko - Macleáya

saarni - Fráxinus

sahrami - Crócus

saippo - Ibéris

saksankirveli - Mýrrhis

saksankumina - Foenículum

salaatti - Lactúca

salkoruusu - Althaéa

salvia - Sálvia

samettikukka - Tagétes

saniainen $\rightarrow$ hirvensarvis., hiuss.,

kelluss., sulkas.

saniaispuu - Alsóphila

sauramo - Ánthemis

selja - Sambúcus

selleri - Ápium

sievikki - Nemóphila

siilikaktus - Echinocáctus

sikuri - Cichórium silkkikukka - Godétia

silkkiäispuu - Mórus

sinappi - Sinápis

sineraaria - Senécio cruéntus

sinilatva - Polemónium

sinililja - Scílla

sinisarjalilja - Agapánthus

sinitähtönen - Agératum

sinivuokko - Hepática

sipuli - Állium

sitruuna - Cítrus

soihtulilja - Kniphófia

soilikki - Streptocárpus

sormustinkukka - Digitális

sorvarinpensas - Euónymus

sukapää - Cynosúrus

sulkasaniainen - Nephrólepis

suopayrtti - Saponária

suppilokukka - Sinníngia

surmaköynnös - Celástrus

syklaami - Cyclámen

sypressi (aito) - Cupréssus; myös

$\rightarrow$ kesäs., vales.

syreeni - Syrínga

syyläkaktus - Mammillária

syyshehku - Dáhlia cultórum

syyslilja - Tritónia

sädekukka - Gaillárdia

sädelatva - Gerbéra

särkynyt sydän - Dicéntra

säänennustaja - Dimorphothéca

taatelipalmu - Phoénix

taikinamarja - Ríbes alpínum

talventähti - Eránthis

talvio - Vínca

tammi - Quércus

tatar - Polýgonum

tikankontti - Cypripédium

tilli - Anéthum

timotei - Phléum praténse

tohvelikukka - Calceolária

tomaatti - Solánum lycopérsicum

torvikukka - Salpiglóssis

tuhatkauno - Achilléa ptármica

fl.pl.

tuhkapensas - Cotoneáster

tuhkaruoho - Senécio cinerària

tuija - Thúja; myös $\rightarrow$ hiba-t.

tulikukka - Verbáscum

tulilatva - Kalánchoë

tuliunikko - Eschschóltzia

tulppaani - Túlipa 


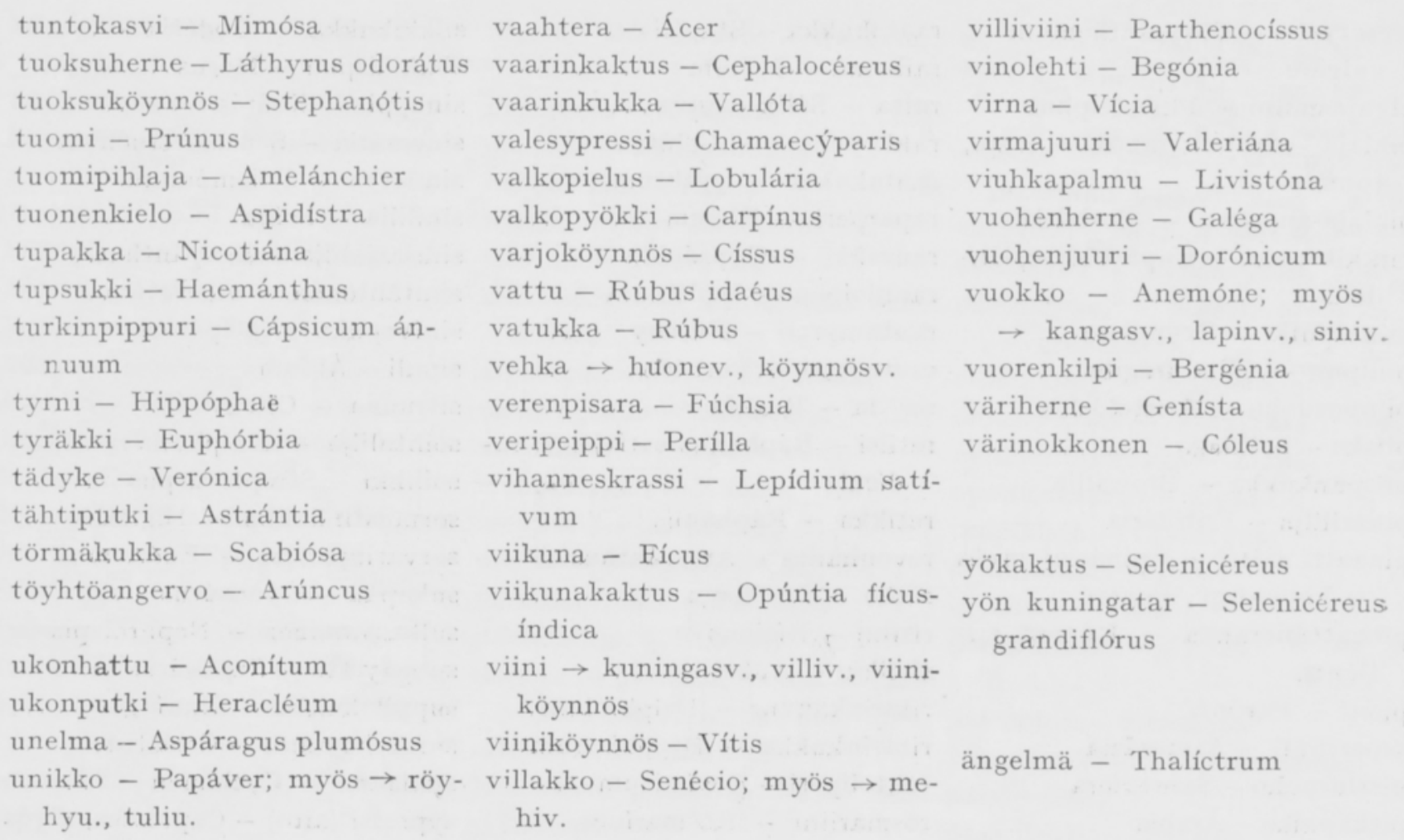

SELITYKSIÄ.

Sulkeissa ovat tieteellisten nimien synonyymit (toisintonimet), suomalaisissa nimissä vuorostaan se osa, jota ei aina tavvitse käytıää.

$$
\begin{aligned}
t . & =\text { forma }=\text { muoto } \\
\text { tl. } p l . & =\text { flore pleno }=\text { kukka tai mykerö kerrottu } \\
\text { ssp. } & =\text { subspecies = alalaji } \\
v . & =\text { varietas = muunnos } \\
\rightarrow & =\text { katso! }
\end{aligned}
$$

